\title{
Resolution of thrombus burden by targeting inflammatory mediators to treat DVT
}

\begin{abstract}
CS
RvD4

treatment was

associated

with reduced

neutrophil and

macrophage

infiltration in

the thrombus
\end{abstract}

At present, effective strategies to resolve persistent venous thrombi and prevent post-thrombotic syndrome, a chronic complication of deep-vein thrombosis (DVT), are limited. Cherpokova and colleagues now describe a novel treatment approach whereby resolvin D4 (RvD4), an endogenously synthesized, specialized proresolving mediator (SPM), administered to mice with DVT significantly reduces thrombus burden and promotes resolution of inflammation. This study is the first to document the endogenous synthesis of SPMs in thrombi in mice with DVT and to establish the role of these SPMs in resolving venous thrombi.

Incomplete or delayed thrombus resolution can promote the development of post-thrombotic syndrome, an often overlooked consequence of DVT. Strategies to resolve venous thrombi and prevent post-thrombotic syndrome are scarce, but SPMs have been suggested as a promising approach for thrombus resolution, given their important role in reducing vessel-wall inflammation and increasing macrophage-mediated thrombus removal. Cherpokova and colleagues sought to "investigate the biosynthesis of SPMs during the progression of DVT in mice and provide evidence that repetitive delivery of selected SPMs reduces [venous thrombosis] burden, a key determinant of [postthrombotic syndrome] development".

Temporal clusters of endogenously synthesized SPMs and proinflammatory lipid mediators were identified in the thrombus during DVT progression in mice with the use of liquid chromatographytandem mass spectrometry. These SPMs and lipid mediators were involved in different phases of venous thrombosis, whereby RvD4, RvD5, RvD6 and

others were associated with the early phase that is characterized by thrombi rich in neutrophils and platelets. Mice subjected to inferior vena cava (IVC) stenosis and treated with a specific mix of four SPMs via intravenous injection showed halted thrombus growth and reduced thrombus length compared with vehicle-treated control mice. "We then focused our attention on a particular SPM of this cluster, RvD4," explains Charlotte Jouvene, a co-first author in the study. "This SPM was previously identified by our laboratory in plasma and serum, and its potent biological actions include a reduction in neutrophil infiltration in peritonitis and Staphylococcus aureus infection, and an increase in macrophage, monocyte and neutrophil phagocytosis."

By day 8 after IVC stenosis, RvD4-treated mice had significantly reduced thrombus length compared with vehicle-treated mice. Furthermore, RvD4 treatment was associated with

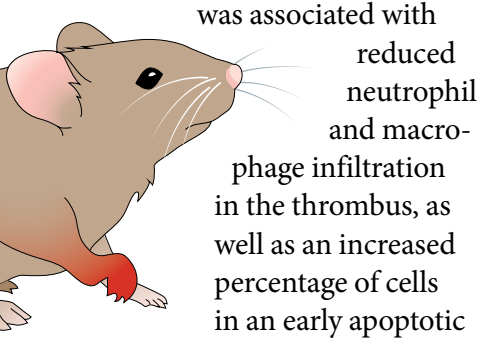

state. Compared with vehicle-treated mice, neutrophils isolated from the blood of RvD4-treated mice were less susceptible to ionomycin-induced release of neutrophil extracellular traps (NETs), a meshwork of chromatin strands studded with granular and cytoplasmic proteins that are critical for the development of thrombosis. Moreover, the mechanism underlying the effects of RvD4 on reducing thrombotic burden is via leukocyte recruitment, apoptosis and NETosis, as opposed to platelet aggregation. "[These findings] support other studies demonstrating novel function of SPMs in modulation of NETosis," comments Jouvene. "Indeed, it has been demonstrated that RvD1treated mice displayed significantly lower levels of citrullinated histone H3, a marker of NETosis, in a model of abdominal aortic aneurysm.” Finally, RvD4 treatment also stimulated the biosynthesis of other D-series resolvins that are involved in the resolution of inflammation.

Taken together, these findings suggest that administration of SPMs, specifically RvD4, can improve the resolution of venous thrombi in a mouse model of IVC stenosis-induced DVT. "Given their anti-inflammatory and resolution-promoting actions and the possibility of being combined with other established treatments, SPMs, such as RvD4, could represent a novel therapeutic approach for thrombo-inflammatory diseases," concludes Jouvene.

Karina Huynh

ORIGINAL ARTICLE Cherpokova, D. et al. Resolvin D4 attenuates the severity of pathological thrombosis in mice. Blood https://doi.org/10.1182/ blood.2018886317 (2019) 